\title{
Manual Muscle Testing
}

National Cancer Institute

\section{Source}

National Cancer Institute. Manual Muscle Testing. NCI Thesaurus. Code C121360.

A graded muscle strength measurement performed as part of a physical examination without the need for specialized equipment. 